\title{
MicroRNA-29b Regulates the Mitochondria-Dependent Apoptotic Pathway by Targeting Bax in Doxorubicin Cardiotoxicity
}

\author{
Xibo Jing ${ }^{a}$ Jingxiao Yang ${ }^{b}$ Lu Jiang ${ }^{c}$ Jianghong Chen ${ }^{a}$ Haiyan Wang ${ }^{b}$ \\ ${ }^{a}$ Department of Cardiology, the Second Affiliated Hospital of Shandong Traditional Chinese Medicine \\ University, Jinan, 'Department of Cardiology, Tangdu Hospital, the Fourth Military University, Xi'an, 'The \\ First Xian Sanatorium of Xinjiang Military Region, Xi'an, China
}

\section{Key Words}

Doxorubicin • Cardiotoxicity • Myocardial apoptosis • MicroRNA-29b • Mitochondria pathway

\begin{abstract}
Background/Aims: Myocardial apoptosis plays an important role in doxorubicin (Dox) cardiotoxicity. MicroRNA-29 (miR-29) is suggested to function as an anti-fibrotic factor with potential therapeutic effects on cardiac fibrosis. However, it has not been shown whether there is an association between miR-29b and myocardial apoptosis. Methods: Male Wistar rats were transfected with miR-29b agomir by local delivery to the myocardium prior to Dox treatment. Rat cardiomyocytes were pretreated with miR-29b mimics or inhibitor followed by Dox incubation in vitro. Cardiac function and underlying mechanisms were evaluated by echocardiography, immunofluorescence, flow cytometry, real-time PCR, and western blotting. Results: Our results revealed that miR-29b is the only member of the miR-29 family that was significantly downregulated in myocardium from Dox-treated rats. Delivery of miR-29b agomir to myocardium resulted in a marked improvement of cardiac function. Terminal deoxynucleotidyl transferase dUTP nick end labeling staining showed that rescue of miR-29b expression inhibited Dox-induced myocardial apoptosis, concomitantly with increased $\mathrm{Bcl}-2$ expression and decreased Bax expression and caspase-3 activity. In vitro, miR-29b overexpression mitigated, whereas inhibition of miR-29b promoted, Dox-induced cardiomyocyte apoptosis. Mechanistically, miR-29b negatively regulated Bax expression by directly targeting the $3^{\prime}$ untranslated region of Bax. In Dox-treated cardiomyocytes, upregulation of miR-29b resulted in a significant decrease in Bax expression, with an increase in $\mathrm{Bcl}-2$ expression, accompanied by inhibition of mitochondrial membrane depolarization, cytochrome $c$ release, and caspase activation. However, inhibition of miR-29b produced the opposite effects by further augmenting the effects of Dox. Conclusions: These data
\end{abstract}

X. Jing and J. Yang contributed equally to this work 
demonstrate that miR-29b prevents Dox-induced myocardial apoptosis through inhibition of the mitochondria-dependent pathway by directly targeting Bax, suggesting that miR-29b is a potential novel therapeutic target for the treatment of Dox cardiotoxicity.

(C) 2018 The Author(s)

Published by S. Karger AG, Basel

\section{Introduction}

Cancer is the second leading cause of death worldwide [1]. Over the past decade, despite survival rates improving due to advances in cancer biology and therapeutics, immunosuppressive and cytotoxic adverse effects still limit the therapeutic use of anticancer drugs [1-3]. Doxorubicin (Dox) is widely used for the treatment of solid tumors and hematologic malignancies [4,5]. Unfortunately, the therapeutic index of this highly potent anti-neoplastic agent is reduced due to its cardiotoxicity, which may lead to severe cardiomyopathy and even cause heart failure and mortality $[1,6]$. It is worth noting that the deterioration of cardiac function is mainly associated with myocardial apoptosis [7, 8]. Being terminally differentiated cells, cardiomyocytes are generally not replicative. Therefore, inhibiting cardiomyocyte apoptosis may be an effective approach to prevent the cardiotoxicity of Dox. However, the molecular mechanisms underlying the Dox-induced apoptosis in cardiomyocytes remain poorly understood.

MicroRNAs (miRs) are a class of endogenous, small (22 nucleotides in length), noncoding RNAs that post-transcriptionally regulate target gene expression by binding to the $3^{\prime}$ untranslated region (3'-UTR) [4]. There is evidence indicating that miRs functionally modulate a variety of physiological or pathological processes [9-11]. More importantly, clinical trials and animal experiments have demonstrated that miRs are potential regulators of the cardiotoxicity of Dox. Recent studies have shown that miR-30e, -208 , and -21 ameliorate Dox-induced cardiomyocyte apoptosis, while miR-320a, $-523-3 p,-140$, and $-146 a$ further enhance apoptosis [4,11-16]. These findings suggest that manipulation of miRs is a promising therapeutic approach. In the current study, we provide the first evidence to our knowledge identifying miR-29b (a member of miR-29 family) as an essential regulator of the mitochondria pathway, cardiomyocyte apoptosis, and Dox cardiotoxicity by directly targeting Bax, a gene critical for the activation of the intrinsic mitochondria pathway. Our data suggest that miR-29b is a potential novel therapeutic target for the treatment of Dox cardiotoxicity during chemotherapy.

\section{Materials and Methods}

\section{Animal model}

Male Wistar rats (250-300 g) were purchased from the Model Animal Research Center of Nanjing University (Nanjing, China). All animal experiments were performed according to the guidelines of the Institutional Animal Care and Use Committee of the Fourth Military University and approved by the Institutional Animal Ethics Committee. miR-29b agomir and mismatched agomir (MA) were designed and obtained from Obio Technology (Shanghai, China). Three days before Dox administration, rats were anesthetized with pentobarbital sodium $(40 \mathrm{mg} / \mathrm{kg}$, intraperitoneally [ip]) and a lateral thoracotomy was performed. miR-29b agomir or MA ( $200 \mathrm{nmol} / \mathrm{kg}$ in a final volume of $200 \mu \mathrm{L})$ was injected into 10 sites at a depth of 1-2 mm in the left ventricular free wall with a 26-gauge syringe as previously described $[17,18]$. After agomir delivery, rats were implanted with a mini osmotic pump (ALZET Model 2002, Cupertino, CA, USA) containing $200 \mu \mathrm{L}$ of either saline (vehicle for Dox) or Dox at a dose of $30 \mathrm{mg} / \mathrm{kg}$ body weight, at a rate of $0.5 \mu \mathrm{L} / \mathrm{h}$ for 14 days.

Real-time PCR

Total RNA from myocardial tissues and cardiomyocytes was isolated using an RNAeasy Kit (Qiagen, Venlo, the Netherlands) according to the manufacturer's instructions. To measure miR-29a, miR-29b, and miR-29c expression, real-time PCR was performed on a 7500 Fast Real-Time PCR System (Applied 


\section{Cellular Physiology Cell Physiol Biochem 2018;48:692-704 \begin{tabular}{l|l} 
and Biochemistry Published online: July 19, 2018 & $\begin{array}{l}\text { C } 2018 \text { The Author(s). Published by S. Karger AG, Basel } \\
\text { www.karger.com/cpb }\end{array}$
\end{tabular} \\ Jing et al.: MicroRNA-29b Prevents Against Doxorubicin Cardiotoxicity}

Biosystems, Carlsbad, CA, USA) using a TaqMan Micro Assay Kit (Thermo Fisher Scientific Inc., Waltham, MA, USA). To determine the caspase-3 and Bax mRNA expression, total RNA was reverse transcribed using a RevertAid First-Strand cDNA Synthesis Kit (Thermo Fisher Scientific Inc.) and PCR was performed with a Fast SYBR Green Master Mix Kit (Applied Biosystems). The real-time PCR experiment was executed in triplicate and the relative mRNA expression index was normalized to U6 or GAPDH. The primer sequences for real-time PCR are listed in Table 1.
Table 1. The primer used for real-time PCR

\begin{tabular}{lcl}
\hline Genes & \multicolumn{2}{c}{ Primers sequences (5' to 3') } \\
\hline \multirow{2}{*}{ Caspase-3 } & Forward & GTGCTACGATCCACCAGCAT \\
& Reverse & AGACCAGTGCTCACAAGGTG \\
Bax & Forward & TGGCGATGAACTGGACAACA \\
& Reverse & CACGGAAGAAGACCTCTCGG \\
& Forward & TGTGAACGGATTTGGCCGTA \\
GAPDH & Reverse & GATGGTGATGGGTTTCCCGT \\
\hline
\end{tabular}

miR in situ hybridization analysis

The probes for in situ hybridization of miR-29b and U6 were synthesized by Sangon Biotech (Shanghai, China). Tissue microarray slides $(4 \mu \mathrm{m})$ were prepared, deparaffinized, deproteinized, and pre-hybridized with hybridization buffer without probe. The hybridization was performed overnight in hybridization buffer with digoxigenin-labeled (DIG-labeled) probes. After washing, the slides were incubated with alkaline phosphatase-conjugated anti-DIG Fab fragments (1:1500; Roche, Indianapolis, IN, USA) and visualized for color detection.

Cardiomyocyte isolation, culture, transfection, and treatment

Cardiomyocytes were isolated from 1- to 2-day-old neonatal Sprague-Dawley rats using a previously described procedure [19]. Cells were cultured in DMEM supplemented with $10 \%$ fetal bovine serum and antibiotics (streptomycin/penicillin, $100 \mu \mathrm{g} / \mathrm{mL}$; all from Invitrogen, Carlsbad, CA, USA) and maintained at $37^{\circ} \mathrm{C}$ and $5 \% \mathrm{CO}_{2}$. miR-29b mimics, miR-29b inhibitor, mimics negative control, and inhibitor negative control were obtained from RiboBio (Guangzhou, China). Cardiomyocytes were starved in serum-free medium for $24 \mathrm{~h}$, and then transfected with miR-29b mimics ( $60 \mathrm{nmol} / \mathrm{L})$, miR-29b inhibitor $(60 \mathrm{nmol} / \mathrm{L})$, or their negative controls using Lipofectamine 2000 (Invitrogen) according to the manufacturer's instructions. Forty-eight hours after transfection, cells were subsequently treated with Dox (10 $\mu \mathrm{mol} / \mathrm{L})$ for another $24 \mathrm{~h}$.

Evans blue dye uptake

Evans blue dye (EBD; Sigma-Aldrich, St. Louis, MO, USA) was dissolved in phosphate buffered saline (PBS; $10 \mathrm{mg} / \mathrm{mL}$ ) and injected ip into rats $(100 \mu \mathrm{g} / \mathrm{g}$ body weight) $24 \mathrm{~h}$ before sacrifice. The ventricular myocardium was harvested and sliced into $8-\mu \mathrm{m}$-thick frozen sections. Immunofluorescence staining was performed with anti- $\alpha$-actinin antibody (Santa Cruz Biotechnology, Dallas, TX, USA; 1:100), and the sections were observed using a confocal microscope (FV1000, Olympus, Tokyo, Japan).

\section{Biochemical assay}

At the end of the animal experiment, blood samples were collected from the abdominal vena cava and centrifuged for $10 \mathrm{~min}$ at $3000 \times \mathrm{g}$ to obtain serum. Cardiomyocytes were treated with miR-29b mimics or miR-29 inhibitor before Dox incubation. Then cell medium was harvested, centrifuged, and diluted. Lactate dehydrogenase (LDH) release was measured by the CytoTox 96 Non-Radioactive Cytotoxicity Assay kit (Promega, Madison, WI, USA). Cardiac troponin T (cTnT) level in serum was determined by an enzyme-linked immunosorbent assay kit (MLBIO Biotechnology, Co., Ltd., Shanghai, China). All assays were performed according to the manufacturers' protocols.

\section{Echocardiography}

Cardiac function was evaluated by echocardiographic analysis using a high-resolution ultrasound imaging system (Vevo770; VisualSonics, Toronto, ON, Canada). At the end of animal experiments, left ventricular end-diastolic posterior wall thickness, left ventricular end-diastolic diameter, left ventricular end-systolic diameter, left ventricular ejection fraction, and left ventricular fractional shortening percentage were measured with the accompanying software. 


\section{Cellular Physiology Cell Physiol Biochem 2018;48:692-704 \begin{tabular}{l|l} 
and Biochemistry Published online: July 19, 2018 & $\begin{array}{l}\text { D) } 2018 \text { The Author(s). Published by S. Karger AG, Basel } \\
\text { www.karger.com/cpb }\end{array}$ \\
\hline
\end{tabular}}

Jing et al.: MicroRNA-29b Prevents Against Doxorubicin Cardiotoxicity

\section{Terminal deoxynucleotidyl transferase dUTP nick end labeling staining}

Cell apoptosis was measured by performing a terminal deoxynucleotidyl transferase dUTP nick end labeling (TUNEL) assay using an In Situ Cell Death Detection kit (Roche, Mannheim, Germany). The frozen sections were incubated with TUNEL Reaction Mixture at $37^{\circ} \mathrm{C}$ for $60 \mathrm{~min}$. Cardiomyocytes were fixed with $4 \%$ paraformaldehyde, permeabilized with $0.1 \%$ Triton X-100, and incubated with TUNEL Reaction Mixture for $60 \mathrm{~min}$ at $37^{\circ} \mathrm{C}$. The nuclei were counterstained with DAPI. Apoptotic cells were observed with a confocal microscope (FV1000). The percentage of TUNEL-positive cells was expressed as a ratio of stained apoptotic cells to total cell number.

\section{Western blotting}

The left ventricular anterior wall or cardiomyocytes were lysed by RIPA lysis buffer (Beyotime Institute of Biotechnology, Shanghai, China) containing protease inhibitor cocktail (Thermo Fisher Scientific Inc.). Intact mitochondria were isolated using a Mitochondria Isolation Kit (Thermo Fisher Scientific Inc.) according to the manufacturer's protocol. Protein concentration was determined using a Bradford assay (Bio-Rad Laboratories, Hercules, CA, USA). Proteins were resolved by sodium dodecyl sulfate polyacrylamide gel electrophoresis gels and transferred onto polyvinylidene fluoride membranes (Millipore, Burlington, MA, USA). Thereafter, the membranes were blocked in $5 \%$ non-fat milk and then incubated with primary antibodies against Bcl-2 (1:1000), Bax (1:1000), cytochrome $c(1: 500)$, caspase-9 (1:1000), caspase-3 (1:500), and PARP (all from Cell Signaling Technology, Danvers, MA, USA); and $\beta$-actin (1:2000) and Cox IV (1:1000) (both Santa Cruz Biotechnology). After incubation with horseradish peroxidase-conjugated secondary antibodies (Beyotime Institute of Biotechnology) for $1 \mathrm{~h}$ at room temperature, blots were visualized by an enhanced chemiluminescence system (Thermo Fisher Scientific Inc.). The intensity of bands was digitally quantified using densitometric analysis software (ImageJ 1.57, NIH, Bethesda, MD, USA).

\section{Caspase-3 activity assay}

Caspase-3 activity in myocardial tissue homogenates was measured using a colorimetric assay kit (Beyotime Institute of Biotechnology) according to the manufacturer's protocol.

\section{Cell viability assay}

The viability of cardiomyocytes was detected using the Cell Counting Kit-8 (CCK-8; Dojindo, Tokyo, Japan). The cells were seeded in 96-well plates at a density of $10^{3}$ cells per well. After the cell treatment described above, CCK-8 reagent was added to the cells for $4 \mathrm{~h}$ at $37^{\circ} \mathrm{C}$ and $5 \% \mathrm{CO}_{2}$. The absorbance was measured at a wavelength of $490 \mathrm{~nm}$ using a microplate reader (Bio-Tek, Winooski, VT, USA).

\section{Apoptosis analysis by flow cytometry}

Cardiomyocyte apoptosis was evaluated using the FITC-Annexin V Apoptosis Detection Kit (Beyotime Institute of Biotechnology) by flow cytometry according to the manufacturer's instructions. Cells were harvested for Annexin V-FITC and propidium iodide (PI) staining for $15 \mathrm{~min}$ at room temperature in the dark. The apoptotic cells were counted by flow cytometry (Accuri C6, BD Biosciences, San Jose, CA, USA).

\section{Luciferase reporter assay}

The wild-type rat Bax 3'-UTR (GenBank ID: NM_017059.2; hereafter referred to as the Bax 3'-UTR) was subcloned into the multiple cloning site of the pmirGLO Dual-Luciferase miRNA Target Expression Vector (Promega). The mutant 3 '-UTR of the Bax gene was generated by substitution of $5 \mathrm{bp}$ from the seed region of miR-29b. Cardiomyocytes were seeded in 96-well plates and co-transfected with luciferase reporter and miR-29b mimics or miR-29b inhibitor using Lipofectamine 2000. The cells were harvested $48 \mathrm{~h}$ later and the luciferase activity was assessed using the Dual-Luciferase Reporter Assay System (Promega).

\section{Mitochondrial membrane potential assay}

Mitochondrial membrane potential (MMP) was measured by 5, 5',6, 6'-tetrachloro-1, 1',3, $3^{\prime}$-tetraethyl-benza-midazolocarbocyanin iodide (JC-1, Invitrogen) fluorescence staining, an indicator of apoptosis. In healthy cells, JC-1 exhibits potential-dependent accumulation in mitochondria, indicated by red fluorescence. In apoptotic cells, the MMP is disrupted and the dye is released into the cytoplasm as a 
monomer and emits green fluorescence. The ratio of red/green fluorescence intensity indicates the change in MMP. Cardiomyocytes were incubated with JC- 1 working solution in the dark at $37^{\circ} \mathrm{C}$ for $15 \mathrm{~min}$. After washing with PBS twice, the fluorescence was measured using a confocal microscope (FV1000).

\section{Statistical analysis}

All data are presented as the mean \pm standard error of the mean and were compared by two-tailed Student's $t$ test or one-way analysis of variance, followed by Bonferroni's multiple-comparison test. Statistical analysis was performed using SPSS 18.0 software (SPSS Inc., Chicago, IL, USA). $P<0.05$ was considered statistically significant.

\section{Results}

miR-29b expression in myocardium is regulated by Dox

The miR-29 family comprises 3 miRs: miR-29a, miR-29b, and miR-29c. We first examined the expression pattern of the miR-29 family in myocardium. Real-time PCR results showed that the expression of miR-29b was approximately 4-fold higher than that of miR-29a and 8-fold higher than that of miR-29c (Fig. 1A). miR-29a and miR-29c expression in myocardium was not significantly influenced by Dox administration (Fig. 1B and C). Interestingly, with regard to miR-29b expression, Dox induced its downregulation in rat myocardium in a timedependent manner (Fig. 1D). Further in situ hybridization analysis with the DIG-labeled miR$29 \mathrm{~b}$ probe also revealed decreased expression of miR-29b in myocardium of Dox-treated rats compared with vehicle-treated rats (Fig. 1E). To further support the in vivo results, rat cardiomyocytes were treated with different concentrations of Dox and miR-29b expression was determined. Compared with the vehicle group, miR-29b expression was significantly decreased by exposure to 1 or $10 \mu \mathrm{mol} / \mathrm{L}$ Dox in cardiomyocytes (Fig. 1F). These data suggest that miR-29b is the dominant member of the miR-29 family expressed in myocardium and that its expression is reduced in response to Dox-induced cardiotoxicity.

Fig. 1. Decreased expression of miR-29b in myocardium of Doxtreated rats. (A) Real-time PCR analysis of miR-29a, miR-29b and miR-29c in myocardium. (B and C) Rats were treated with doxorubicin (Dox; $30 \mathrm{mg} / \mathrm{kg}$ of body weight) for 14 days. The expression of miR-29a (B) and miR-29c (C) in myocardium was examined. (D) The expression of miR-29b in myocardium was determined after 1, 5, 10 and 14 days of Dox treatment. ${ }^{* *} \mathrm{P}<0.01$ vs. corresponding vehicle rats, $n=5$ in each group. (E) Representative ISH staining images showing decreased miR-29b expression in myocardium after Dox treatment for 14 days compared with vehicle group. The U6 was detected by ISH as an internal control. (F) Cardiomyocytes were treated with different concentrations of Dox $(0.1,1$ or $10 \mu \mathrm{mol} / \mathrm{L})$ for $24 \mathrm{~h}$, the expression of miR-29b was determined by realtime PCR. ${ }^{*} \mathrm{P}<0.01$ vs. control $(0 \mu \mathrm{mol} / \mathrm{L}), \mathrm{n}=6$.

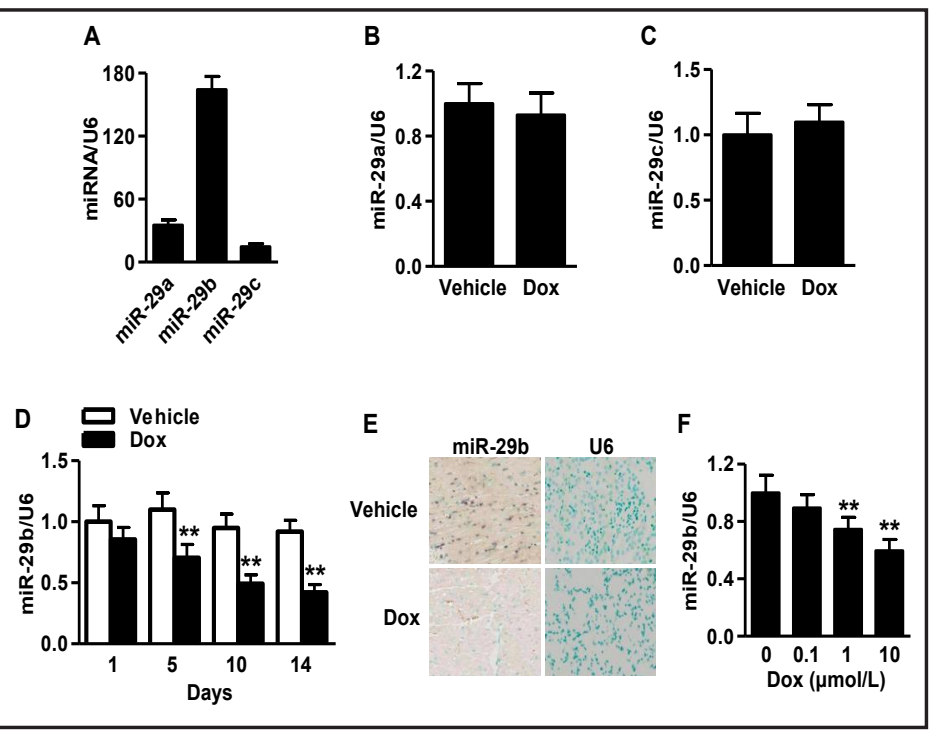


Fig. 2. miR-29b upregulation ameliorates Dox-induced cardiotoxicity. (A) Rats were pretreated with mismatched agomir (MA) or miR-29b agomir, followed by infusion with Dox $(30 \mathrm{mg} / \mathrm{kg}$ of body weight) for 14 days. Representative images of myocardial Evans bule dye (EBD) uptake (red) and viable cardiomyocytes labeled by $\alpha$-actinin (green). The nuclei were counterstained with DAPI (blue). (B) Quantification of EBD uptake. $n=6$ in each group. (C) Lactate dehydrogenase (LDH) (C) and cardiac troponin $\mathrm{T}$ (cTnT) level (D) in serum were measured by a commercial kit. $n=12$ in each group. (E) Representative photograph of heart function. (F-J) Echocardiographic analysis of left ventricular end-diastolic posterior wall thickness (LVPWth) (F), left ventricular

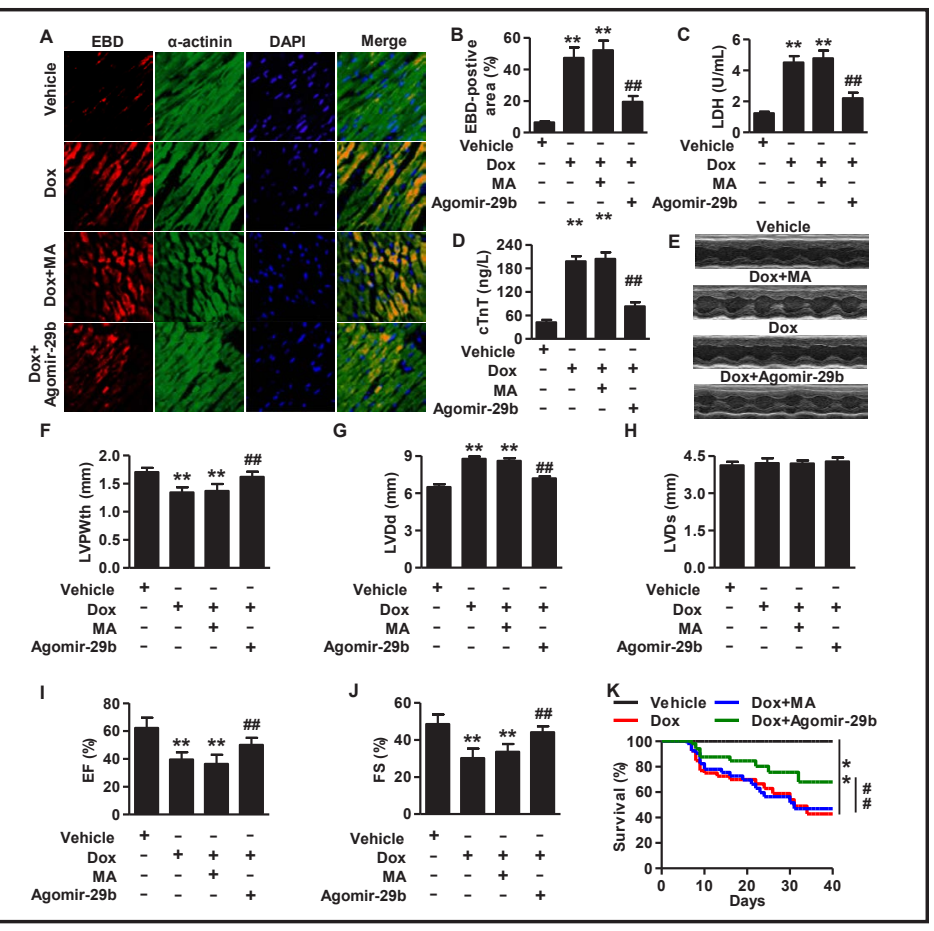
end-diastolic diameter (LVDd) (G), left ventricular end-systolic diameter (LVDs) (H), left ventricular ejection fraction (EF) (I) and left ventricular fractional shortening (FS) (J). n=11 in each group. (K) Survival curves were recorded until day 40 after the start of Dox treatment. $\mathrm{n}=20$ in each group. ${ }^{* *} \mathrm{P}<0.01$ vs. Vehicle; \#\#P<0.01 vs. Dox.

\section{Overexpression of miR-29b blocks Dox-induced myocardial injury}

To investigate the role of miR-29b in the sequelae of Dox-induced cardiotoxicity, rats were treated with miR-29b agomir or MA prior to Dox administration. As shown in Fig. 2A, Dox insult resulted in a loss of cardiomyocyte membrane integrity, as evidenced by increased EBD-positive staining. However, miR-29b agomir treatment significantly decreased EBD-positive staining (Fig. 2B). As a marker for cardiac injury, the release of LDH was measured. We observed that the serum LDH and cTnT concentrations in Dox-treated rats were higher than those of vehicle-treated rats, and miR-29b agomir abolished these elevations (Fig. 2C and D). Furthermore, echocardiographic examination showed that Dox treatment displayed changes in left ventricular morphology, characterized by left ventricular end-diastolic posterior wall thinning with increased left ventricular end-diastolic diameter. These parameters were significantly improved by miR-29b overexpression (Fig. 2E-G). No significant differences were observed in left ventricular end-systolic diameter among the 4 experimental groups (Fig. 2H). miR-29b upregulation also inhibited the decrease in ejection fraction and fractional shortening percentage, indicating improved cardiac function (Fig. 2I and J). Additionally, miR-29b agomir-treated rats showed lower Dox-induced mortality (Fig. $2 \mathrm{~K})$.

\section{miR-29b upregulation attenuates Dox-induced myocardial apoptosis}

Based on the protective effect of miR-29b against Dox-induced myocardial injury, we subsequently aimed to investigate the effect of miR-29b upregulation on myocardial apoptosis. Fig. 3A and B showed that the number of TUNEL-positive cells was significantly increased in myocardium of Dox-treated rats, which was diminished by miR-29b agomir but not MA. To understand how miR-29b inhibits myocardial apoptosis, the expression of apoptosisrelated molecules was examined. Dox insult dramatically decreased the expression of the anti-apoptotic protein $\mathrm{Bcl}-2$ and increased the expression of the pro-apoptotic protein Bax in

\section{KARGER}


Fig. 3. Overexpression of miR-29b attenuates Dox-induced myocardial apoptosis. (A) Apoptosis was examined by a terminal deoxynucleotidyl transferase dUTP nick end labeling (TUNEL) staining. Representative TUNEL (green) and $\alpha$-actinin (red) stained photographs of cardiomyocytes in myocardial tissues. The nuclei were counterstained with DAPI (blue). (B) Quantification of TUNEL-positive cells. $n=6$ in each group. (C and D) The protein expressions of Bcl-2 (C) and Bax (D) in myocardium were determined using western blotting analysis. $\mathrm{n}=7$ in each group. (E) Caspase-3 activity in myocardial tissue homogenates was measured by a colorimetric assay kit. (F) The mRNA expression of caspase- 3 was determined by real-time PCR. $\mathrm{n}=6$ in each group. $* * \mathrm{P}<0.01$ vs. Vehicle; \#\# $\mathrm{P}<0.01$ vs. Dox.

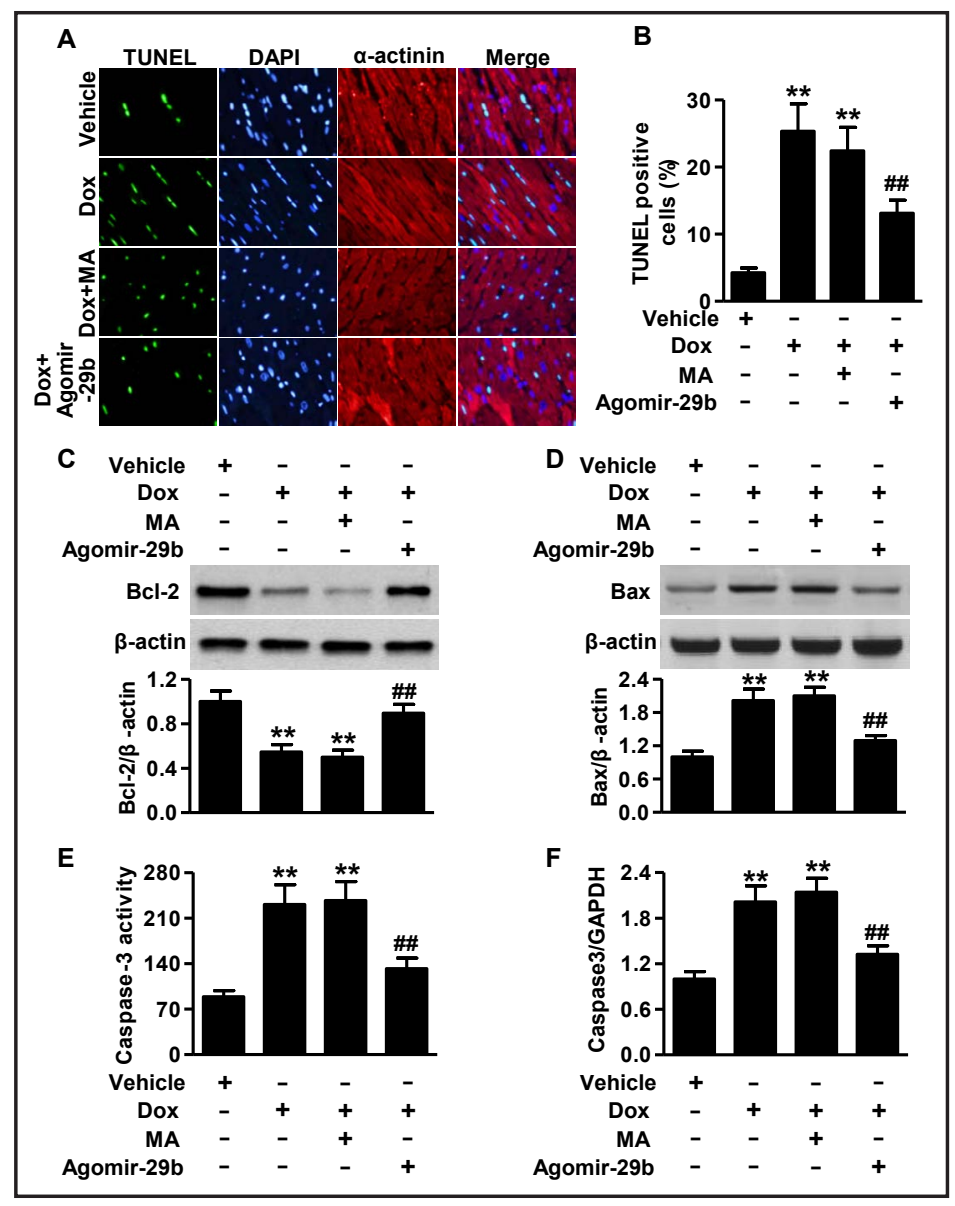

myocardial tissues. However, miR-29b agomir treatment resulted in higher Bcl-2 expression and lower Bax expression than that in Dox-treated rats (Fig. 3C and D). We also measured the changes in caspase-3 mRNA expression and activity. Dox administration increased the mRNA expression and activity of caspase-3. As expected, these elevations were markedly blocked by miR-29b treatment (Fig. 3E and F). These results indicate that the inhibition of apoptosis may contribute to the protective effect of miR-29b against myocardial injury.

miR-29b overexpression mitigates Dox-induced cardiomyocyte apoptosis in vitro

We next tried to confirm whether the anti-apoptotic effects of miR-29b in myocardium also existed in vitro under Dox stimulation. The CCK-8 assay showed that Dox treatment significantly decreased the viability of cardiomyocytes, which was suppressed by miR-29b overexpression induced by miR-29b agomir. By contrast, inhibition of miR-29b by miR$29 \mathrm{~b}$ inhibitor potentiated the decrease in cell viability (Fig. 4A). Similarly, LDH release in cardiomyocytes was also markedly increased after Dox challenge. Overexpression of miR$29 \mathrm{~b}$ suppressed, whereas miR-29b inhibition enhanced, the Dox-induced release of LDH (Fig. 4B). The annexin V-FITC/PI data showed that Dox treatment induced an increase in the apoptotic rate from $11.5 \% \pm 2.3 \%$ to $29.5 \% \pm 3.0 \%$ in cardiomyocytes when compared with the vehicle group. Upregulation of miR-29b decreased the apoptotic cell rate to $17.6 \% \pm$ $2.1 \%$, whereas miR-29b inhibition further increased the apoptotic rate to $43.3 \% \pm 4.9 \%$ (Fig. 4C and D). Similar to the results of flow cytometry, miR-29b upregulation resulted in fewer TUNEL-positive cells compared with Dox treatment alone. However, inhibition of miR-29b showed the opposite effect (Fig. 4E and F). 
Fig. 4. miR-29b mediates cardiomyocyte apoptosis induced by Dox. (A) Cardiomyocytes were transfected with miR-29b mimics (29b-m, $60 \mathrm{nmol} / \mathrm{L})$, miR-29b inhibitor (29b-i, $60 \mathrm{nmol} / \mathrm{L})$ or their negative controls (NC-m or NC-i) for $48 \mathrm{~h}$ prior to Dox $(10 \mu \mathrm{mol} / \mathrm{L})$ incubation for another $24 \mathrm{~h}$. Cell viability was assessed with CCK-8 assay. (B) At the end of the experiment, cell medium was harvested and LDH release was measured. (C) Cardiomyocyte apoptosis was determined by annexin V/PI staining followed by flow cytometry. (D) Quantitative analysis of the percentage of apoptotic cells. (E) TUNEL (green) and DAPI staining (blue) of cardiomyocytes. (F) The percentage of TUNEL-positive cells was calculated. ${ }^{* *} \mathrm{P}<0.01$ vs. Vehicle; \#\#P<0.01 vs. Dox, $\mathrm{n}=6$.

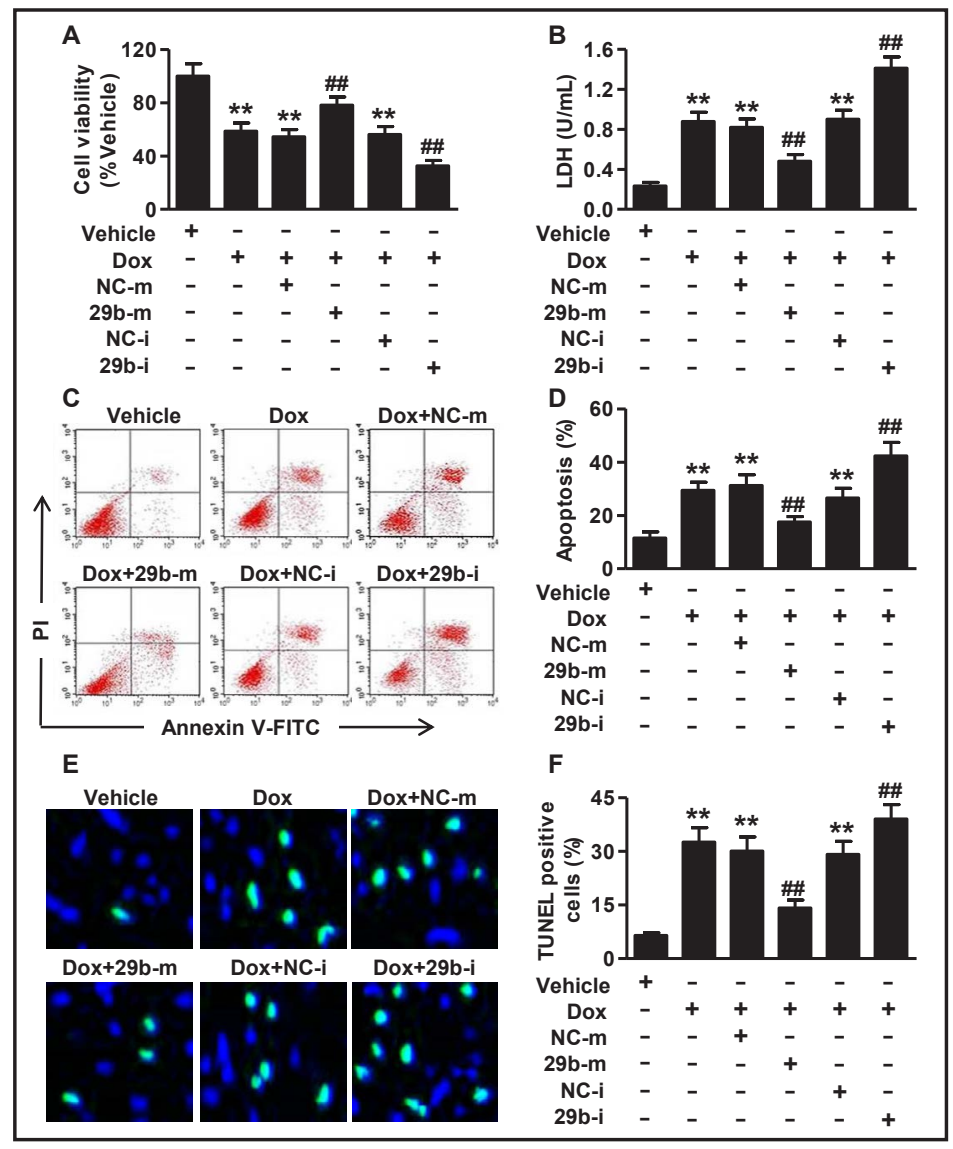

Fig. 5. miR-29b negatively regulates Bax expression. (A) Predicted miR-29b seed matches to the sequence in the 3 '-UTR of Bax. The complementary sequences are shown in green and blue, and the mutated sequences are shown in red. (B and C) Dual luciferase activity assay was performed by cotransfection of luciferase reporter containing Bax 3'-UTR or the mutant one with miR-29b mimics (B)

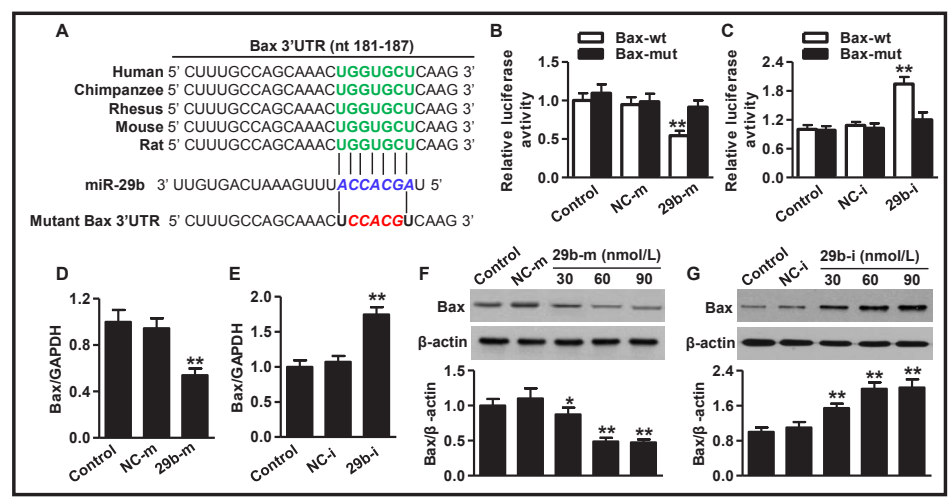
or miR-29b inhibitor (C) in cardiomyocytes. (D and E) Real-time PCR analysis of Bax mRNA expression in cardiomyocytes transfected with miR-29b mimics (D) and miR-29b inhibitor (E) for $48 \mathrm{~h}$. (F and G) The Bax protein expression was determined by western blotting. ${ }^{* *} \mathrm{P}<0.01$ vs. control, $\mathrm{n}=6$.

\section{miR-29b directly targets the Bax 3'-UTR}

Computational mRNA target analysis (www.Targetscan.org) showed that Bax is a potential molecular target of miR-29b. The miR-29b binding site in the Bax $3^{\prime}$-UTR is conserved among human, chimpanzee, rhesus monkey, mouse, and rat (Fig. 5A). To confirm whether miR-29b can decrease Bax expression through direct $3^{\prime}$-UTR interaction, we generated a firefly luciferase reporter plasmid containing the Bax $3^{\prime}$-UTR and performed reporter analysis. By co-transfection of the reporter plasmid with miR-29b mimics, miR$29 \mathrm{~b}$ inhibitor, or their corresponding negative controls, and Bax 3'-UTR or the mutant in 


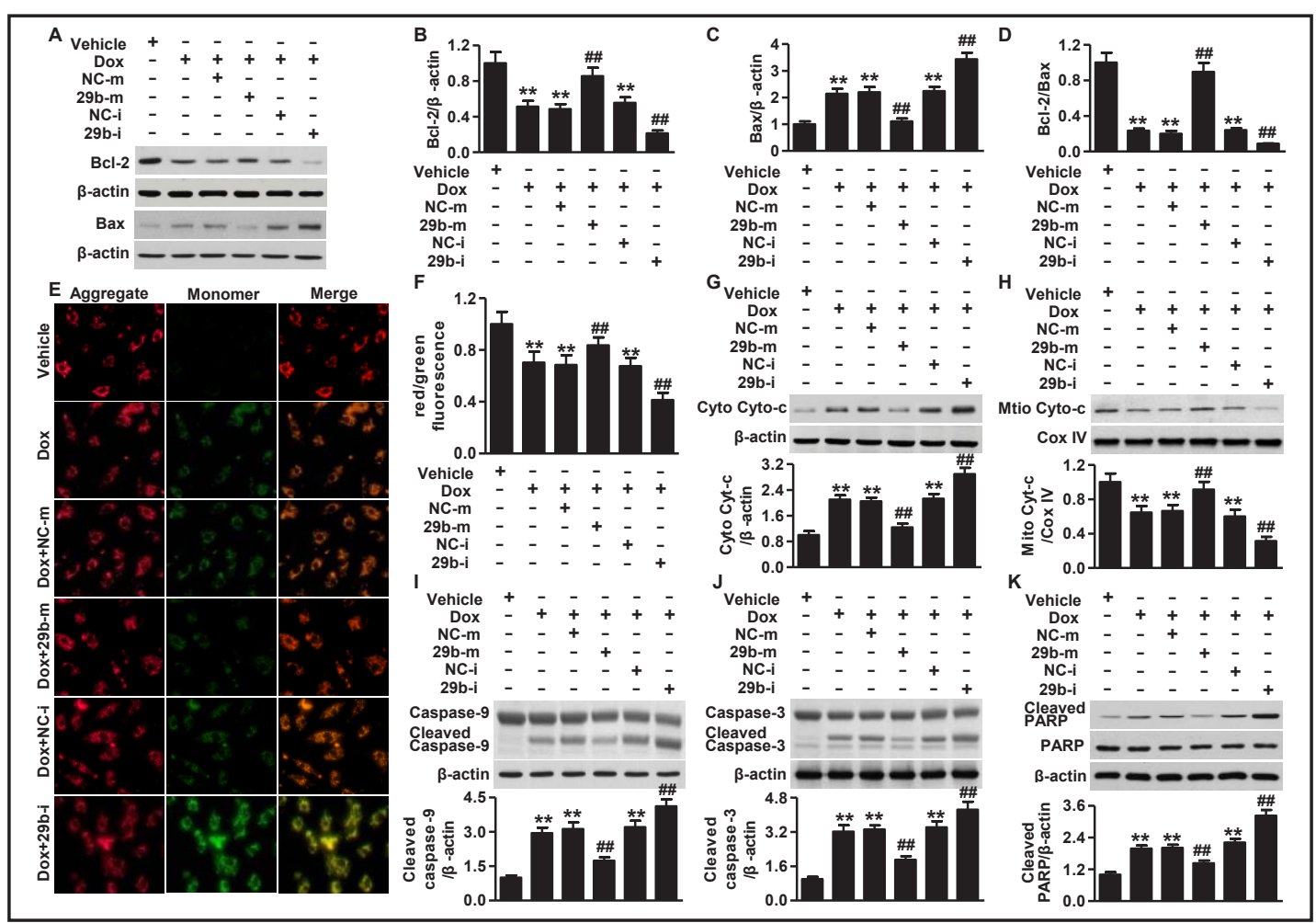

Fig. 6. Mitochondria-dependent pathway is involved in miR-29b-mediated cardiomyocyte apoptosis. (A) Bcl-2 and Bax expression were examined by western blotting. Representative images are shown. (B-D) Densitometric analysis of Bcl-2 (B), Bax (C) and Bcl-2/Bax ratio (D). (E) Mitochondrial membrane potential (MMP) was measured using JC-1 staining. Representative images of JC-1 derived fluorescence in cardiomyocytes. The JC-1 aggregate image was shown red fluorescence; the JC-1 monomer image was shown green fluorescence; the merged image was combined of the red and green images. (F) Quantitative analysis of the ratio of red/green fluorescence. ( $\mathrm{G}$ and $\mathrm{H}$ ) Western blotting analysis of cytochrome c protein expression in the cytoplasm (cyto) (G) and mitochondria (mito) (H). (I-K) The cleaved caspase-9 (I), caspase-3 (J) and PARP $(\mathrm{K})$ were examined by western blotting. ${ }^{* *} \mathrm{P}<0.01$ vs. Vehicle; $\# \# \mathrm{P}<0.01$ vs. Dox, $\mathrm{n}=6$.

cardiomyocytes, the luciferase assay showed that miR-29b overexpression significantly decreased the luciferase activity of the Bax 3'-UTR. However, miR-29b mimics failed to affect the luciferase activity elicited by the reporter carrying the Bax 3'-UTR with the mutant miR$29 \mathrm{~b}$ binding site (Fig. 5B). In contrast, inhibition of miR-29b increased the luciferase activity of the Bax 3'-UTR, but also had no effect on the mutant (Fig. 5C). In agreement with the above observations, miR-29b upregulation effectively decreased the mRNA expression of Bax, whereas downregulation of miR-29b increased Bax mRNA expression in cardiomyocytes (Fig. 5D and E). Furthermore, the protein expression of Bax after miR-29 mimics or inhibitor transfection showed similar changes in mRNA expression (Fig. 5F and G). Taken together, these results demonstrate that Bax is the target gene of miR-29b.

\section{Mitochondria-dependent pathway is involved in miR-29b-mediated cardiomyocyte apoptosis}

Consistent with the results in vivo, Dox treatment for $24 \mathrm{~h}$ obviously decreased Bcl-2 expression and increased Bax expression, resulting in a decrease in the Bcl-2/Bax ratio. Overexpression of miR-29b attenuated the effect of Dox on Bcl-2 and Bax expression and the $\mathrm{Bcl}-2 / \mathrm{Bax}$ ratio. However, miR-29b inhibition further enhanced Bax expression and reduced $\mathrm{Bcl}-2$ expression and the $\mathrm{Bcl}-2 / \mathrm{Bax}$ ratio (Fig. 6A-D). Imbalance between $\mathrm{Bcl}-2$ and Bax expression has been suggested to be associated with mitochondria dysfunction [20]. 
Therefore, we further examined whether miR-29b could influence MMP in cardiomyocytes by JC-1 staining. Confocal microscopy showed that Dox decreased red fluorescence and increased green fluorescence, leading to a decrease in the red/green fluorescence intensity ratio as compared with the vehicle group, indicating a loss of MMP. Inhibition of miR-29b markedly enhanced the Dox-induced loss of MMP, and inverse results were obtained in cells treated with miR-29b agomir (Fig. 6E and F). It has been documented that the increase in MMP induces cytochrome $c$ release from mitochondria to the cytoplasm and subsequently results in caspase cascade activation [21]. Western blotting showed that the translocation of cytochrome $c$ from mitochondria to cytoplasm was dramatically increased after Dox challenge, which was blocked by miR-29b agomir (Fig. 6G). In contrast, miR-29b inhibition further promoted the translocation of cytochrome $c$ induced by Dox (Fig. 6G). In parallel with this, Dox treatment significantly increased caspase-9, caspase-3, and PARP cleavage. Overexpression of miR-29b inhibited, whereas miR-29b inhibition further increased, the activation of caspase-9, caspase-3, and PARP (Fig. 6I-K). Collectively, these data indicate that miR-29b attenuates Dox-induced cardiomyocyte apoptosis at least in part through inhibiting the mitochondria-dependent pathway.

\section{Discussion}

Cardiomyocyte apoptosis is a major feature of Dox cardiotoxicity. Herein, we provide evidence that miR-29b is downregulated in response to Dox treatment and attenuates cardiotoxicity associated with cardiomyocyte apoptosis. In support of this evidence, using gain- and loss-of-function approaches, we found that miR-29b inhibits the mitochondriadependent apoptotic pathway by directly targeting Bax, an effect that reduces the Bax/Bcl-2 ratio and cytochrome $c$ release, and attenuates caspase cascade activation. With regard to these results, our study identifies miR-29b as a critical regulator of cardiomyocyte apoptosis induced by Dox.

miR-29b belongs to the miR-29 family, which includes 3 mature miRNAs: miR-29a, miR29b (miR-29b-1 and miR-29b-2), and miR-29c. These mature sequences are transcribed from two different gene clusters located on two different chromosomes, respectively [22, 23]. miR-29b-1 and miR-29b-2 (herein referred to as miR-29b) share the same mature sequence, while miR-29a and miR-29c differ by one nucleotide [24]. This miR family has been well recognized as an important regulator of collagen expression and heart fibrosis $[25,26]$. More recent studies revealed that miR-29a and miR-29b regulate apoptosis by targeting different genes $[27,28]$. Altered expression of miR-29b has been detected in multiple tumors and leukemia [28, 29], raising the intriguing possibility that aberrant miR-29b expression and/or function may be associated with dysfunction of the apoptosisrelated pathway. Recent studies have demonstrated that miR-29a and miR-29c are involved in neuronal apoptosis and the serum levels of these miRs are negatively correlated with Parkinson's disease severity [24, 30, 31]. Han et al. reported that miR-29a regulated high glucose-induced myocardial cell apoptosis by targeting IGF-1 [32]. Swimming training exercise can induce miR-29c upregulation, which is related to a significant reduction in collagen gene expression and improvement of cardiac function [25]. In the present study, we found that miR-29b is highly expressed in myocardium compared with miR-29a and miR29c. Interestingly, its expression was dramatically decreased after Dox challenge in vivo and in vitro, indicating a potential role of miR-29b in Dox cardiotoxicity. By contrast, Leger et al. showed that miR-29b was upregulated in plasma of children and young adults 6 to $24 \mathrm{~h}$ after anthracycline administration [3]. Presumably, the discrepancy is probably related to (1) miR$29 \mathrm{~b}$ expression possibly having different roles in cardiotoxicity in different samples (plasma or myocardium) even under the same stimulation, and (2) the extent of cardiotoxicity as well as the change in miR-29b possibly being differentially induced by anthracycline compounds with different time periods. 
Dox is a highly potent chemotherapeutic agent. However, its potential usage is largely limited due to its cardiotoxicity, which was reported to be fatal in some cases $[2,6]$. Dox cardiotoxicity features a reduction of ejection fraction and thinning and dilatation of the ventricular wall [8]. Here, we found a marked reduction in left ventricular end-diastolic posterior wall thickness, ejection fraction, and fractional shortening percentage in Dox-treated rats, concomitantly with increased cardiomyocyte membrane permeability and serum LDH concentration. This was consistent with previous studies in several animal models $[8,19,33]$. However, upregulation of miR-29b was effective in improving cardiac function. Also, miR$29 \mathrm{~b}$ agomir-treated rats significantly decreased the susceptibility to Dox-induced mortality. These data suggest that miR-29b can prevent Dox-induced cardiotoxicity. It is worth noting that inhibition of miR-29b led to an increase in collage gene expression and a subsequent decrease in aneurysm expansion [23, 34, 35]. In contrast, miR-29b overexpression increased abdominal aortic aneurysm (AAA) expansion and the aortic rupture rate [35]. These studies indicate a protective role of miR-29b inhibition during AAA development. However, given the significant role of miR-29b in the reduction of collagen expression, endogenous miR-29b diminished many fibrotic events, particularly in the heart, and addition of exogenous miR$29 \mathrm{~b}$ could also inhibit ventricular compliance development [9]. Thus, precise modulation of miR-29b expression seems to be of great importance in specific local tissues and different pathological processes, especially in cardiovascular diseases.

It has been suggested that Dox cardiotoxicity is well characterized by myocardial apoptosis, leading to loss of myocytes and deterioration of heart function $[7,8,16]$. miR29 is an important regulator in cardiac physiology that diminishes extracellular matrix deposition and heart fibrosis $[25,26]$. However, until now it was unclear whether there is a link between miR-29b and apoptosis in cardiomyocytes. In the present study, we observed a significant increase in myocardial apoptosis in Dox-treated rats, which was inhibited by miR-29b upregulation. This was further confirmed by overexpression of miR-29b using a miR-29b mimics in vitro, while miR-29b inhibition further promoted cardiomyocyte apoptosis. Several biochemical pathways, including disruption of intracellular calcium homeostasis, increased generation of reactive oxygen species, inhibition of anti-apoptotic proteins, and induction of pro-apoptotic proteins, have been shown to be associated with Dox-induced myocardial apoptosis and cardiotoxicity [5]. These cellular events are considered to induce apoptosis via the intrinsic pathway. The Bcl-2 family plays a key role in the apoptotic machinery and a variety of upstream survival signals. Bcl-2 is one of the important anti-apoptotic proteins, whereas Bax promotes apoptosis [20]. Thus, the Bcl-2/ Bax ratio determines the fate of cells [21,27]. Moreover, caspase-3 has been confirmed as a dominant executor in the intrinsic pathway of apoptosis [21]. Here, our data demonstrated that miR-29b agomir treatment resulted in a marked increase in Bcl-2 and a decrease in Bax as well as downregulation of caspase activity and mRNA expression. It has been previously reported that miR-29a suppresses Bcl-2 and Mcl-1 expression and increases hepatocellular carcinoma cell, cholangiocarcinoma cell, and neuronal apoptosis [10, 22, 27], suggesting an interaction between miR-29b and Bcl-2 or Mcl-1 in various cell types. These differences may be attributable to different mechanisms involved in and related to the regulation of specific cellular functions and disease states.

We next investigated the mechanism that allows miR-29b to ameliorate Dox-induced cardiomyocyte apoptosis. Our data showed that Bax is a novel target of miR-29b. miR-29b targeted the Bax 3'-UTR to decrease Bax expression, while inhibition of miR-29b significantly increased Bax expression. By binding to pore proteins on mitochondrial membranes, Bax can affect MMP, which leads to mitochondrial membrane depolarization and cytochrome $c$ release from mitochondria to the cytoplasm [20]. The released cytochrome $c$ combines with caspase- 9 to form a complex and activates the caspase cascade, in which caspase- 3 plays a dominant role $[21,36]$. Upon DNA cleavage by caspase-3 activation, PARP depletes ATP in order to repair damaged DNA and maintain cell viability [5]. Depletion of ATP can result in cell lysis and death [36]. In this study, we found that overexpression of miR-29b in cardiomyocytes restored the loss of MMP induced by Dox, and decreased the release of

\section{KARGER}


cytochrome $c$ and cleaved caspase-3, caspase-9, and PARP. Inhibition of miR-29b, however, produced the opposite effects. These findings suggest that miR-29b upregulation inhibits the mitochondria-dependent pathway by directly targeting Bax.

In conclusion, this study demonstrates that, in addition to regulating heart fibrosis, miR$29 \mathrm{~b}$ also has an important role in myocardial apoptosis. We provide the first evidence to our knowledge of the anti-apoptotic property of miR-29b in cardiomyocytes, which offers a rational therapeutic strategy to combat cardiotoxicity and even heart failure during Dox treatment.

\section{Acknowledgements}

This work was supported by Key Science and Technology Program of Shanxi Province (No. 2014K11-03-03-04).

\section{Disclosure Statement}

The authors have declared that no competing interests exist.

\section{References}

1 Zagar TM, Cardinale DM, Marks LB: Breast cancer therapy-associated cardiovascular disease. Nat Rev Clin Oncol 2016;13:172-184.

-2 Rochette L, Guenancia C, Gudjoncik A, Hachet O, Zeller M, Cottin Y, Vergely C: Anthracyclines/trastuzumab: new aspects of cardiotoxicity and molecular mechanisms. Trends Pharmacol Sci 2015;36:326-348.

-3 Leger KJ, Leonard D, Nielson D, de Lemos JA, Mammen PP, Winick NJ: Circulating microRNAs: Potential Markers of Cardiotoxicity in Children and Young Adults Treated With Anthracycline Chemotherapy. J Am Heart Assoc 2017;6:

4 Yin Z, Zhao Y, Li H, Yan M, Zhou L, Chen C, Wang DW: miR-320a mediates doxorubicin-induced cardiotoxicity by targeting VEGF signal pathway. Aging (Albany NY) 2016;8:192-207.

5 L'Ecuyer T, Sanjeev S, Thomas R, Novak R, Das L, Campbell W, Heide RV: DNA damage is an early event in doxorubicin-induced cardiac myocyte death. Am J Physiol Heart Circ Physiol 2006;291:H1273-1280.

6 Zhang S, Liu X, Bawa-Khalfe T, Lu LS, Lyu YL, Liu LF, Yeh ET: Identification of the molecular basis of doxorubicin-induced cardiotoxicity. Nat Med 2012;18:1639-1642.

7 Nitobe J, Yamaguchi S, Okuyama M, Nozaki N, Sata M, Miyamoto T, Takeishi Y, Kubota I, Tomoike H: Reactive oxygen species regulate FLICE inhibitory protein (FLIP) and susceptibility to Fas-mediated apoptosis in cardiac myocytes. Cardiovasc Res 2003;57:119-128.

-8 Shoukry HS, Ammar HI, Rashed LA, Zikri MB, Shamaa AA, Abou Elfadl SG, Rub EA, Saravanan S, Dhingra S: Prophylactic supplementation of resveratrol is more effective than its therapeutic use against doxorubicin induced cardiotoxicity. PLoS One 2017;12:e0181535.

-9 Habibi P, Alihemmati A, Nasirzadeh M, Yousefi H, Habibi M, Ahmadiasl N: Involvement of microRNA-133 and -29 in cardiac disturbances in diabetic ovariectomized rats. Iran J Basic Med Sci 2016;19:1177-1185.

$>10$ Liu XJ, Zheng XP, Zhang R, Guo YL, Wang JH: Combinatorial effects of miR-20a and miR-29b on neuronal apoptosis induced by spinal cord injury. Int J Clin Exp Pathol 2015;8:3811-3818.

11 Lai L, Chen J, Wang N, Zhu G, Duan X, Ling F: MiRNA-30e mediated cardioprotection of ACE2 in rats with Doxorubicin-induced heart failure through inhibiting cardiomyocytes autophagy. Life Sci 2017;169:69-75.

12 Tony H, Yu K, Qiutang Z: MicroRNA-208a Silencing Attenuates Doxorubicin Induced Myocyte Apoptosis and Cardiac Dysfunction. Oxid Med Cell Longev 2015;2015:597032.

13 Tong Z, Jiang B, Wu Y, Liu Y, Li Y, Gao M, Jiang Y, Lv Q, Xiao X: MiR-21 Protected Cardiomyocytes against Doxorubicin-Induced Apoptosis by Targeting BTG2 Int J Mol Sci 2015;16:14511-14525.

14 Wang JX, Zhang XJ, Feng C, Sun T, Wang K, Wang Y, Zhou LY, Li PF: MicroRNA-532-3p regulates mitochondrial fission through targeting apoptosis repressor with caspase recruitment domain in 


\section{Cellular Physiology Cell Physiol Biochem 2018;48:692-704 \begin{tabular}{l|l|l|}
\hline DOI: 10.1159/000491896 & $\begin{array}{l}\text { C) } 2018 \text { The Author(s). Published by S. Karger AG, Basel } \\
\text { www.karger.com/cpb }\end{array}$
\end{tabular}}

Jing et al.: MicroRNA-29b Prevents Against Doxorubicin Cardiotoxicity

doxorubicin cardiotoxicity. Cell Death Dis 2015;6:e1677.

15 Li J, Li Y, Jiao J, Wang J, Li Y, Qin D, Li P: Mitofusin 1 is negatively regulated by microRNA 140 in cardiomyocyte apoptosis. Mol Cell Biol 2014;34:1788-1799.

16 Horie T, Ono K, Nishi H, Nagao K, Kinoshita M, Watanabe S, Kuwabara Y, Nakashima Y, Takanabe-Mori R, Nishi E, Hasegawa K, Kita T, Kimura T: Acute doxorubicin cardiotoxicity is associated with miR-146ainduced inhibition of the neuregulin-ErbB pathway. Cardiovasc Res 2010;87:656-664.

17 Kitsis RN, Buttrick PM, McNally EM, Kaplan ML, Leinwand LA: Hormonal modulation of a gene injected into rat heart in vivo. Proc Natl Acad Sci U S A 1991;88:4138-4142.

18 Yang B, Lin H, Xiao J, Lu Y, Luo X, Li B, Zhang Y, Xu C, Bai Y, Wang H, Chen G, Wang Z: The muscle-specific microRNA miR-1 regulates cardiac arrhythmogenic potential by targeting GJA1 and KCNJ2 Nat Med 2007;13:486-491.

19 Sun C, Liu H, Guo J, Yu Y, Yang D, He F, Du Z: MicroRNA-98 negatively regulates myocardial infarctioninduced apoptosis by down-regulating Fas and caspase-3 Sci Rep 2017;7:7460.

20 Kuwana T, Newmeyer DD: Bcl-2-family proteins and the role of mitochondria in apoptosis. Curr Opin Cell Biol 2003;15:691-699.

21 Ott M, Gogvadze V, Orrenius S, Zhivotovsky B: Mitochondria, oxidative stress and cell death. Apoptosis 2007;12:913-922.

22 Mott JL, Kobayashi S, Bronk SF, Gores GJ: mir-29 regulates Mcl-1 protein expression and apoptosis. Oncogene 2007;26:6133-6140.

23 Merk DR, Chin JT, Dake BA, Maegdefessel L, Miller MO, Kimura N, Tsao PS, Iosef C, Berry GJ, Mohr FW, Spin JM, Alvira CM, Robbins RC, Fischbein MP: miR-29b participates in early aneurysm development in Marfan syndrome. Circ Res 2012;110:312-324.

24 Bai X, Tang Y, Yu M, Wu L, Liu F, Ni J, Wang Z, Wang J, Fei J, Wang W, Huang F, Wang J: Downregulation of blood serum microRNA 29 family in patients with Parkinson's disease. Sci Rep 2017;7:5411.

25 Melo SF, Fernandes T, Barauna VG, Matos KC, Santos AA, Tucci PJ, Oliveira EM: Expression of MicroRNA-29 and Collagen in Cardiac Muscle after Swimming Training in Myocardial-Infarcted Rats. Cell Physiol Biochem 2014;33:657-669.

26 van Rooij E, Sutherland LB, Thatcher JE, DiMaio JM, Naseem RH, Marshall WS, Hill JA, Olson EN: Dysregulation of microRNAs after myocardial infarction reveals a role of miR-29 in cardiac fibrosis. Proc Natl Acad Sci U S A 2008;105:13027-13032.

27 Xiong Y, Fang JH, Yun JP, Yang J, Zhang Y, Jia WH, Zhuang SM: Effects of microRNA-29 on apoptosis, tumorigenicity, and prognosis of hepatocellular carcinoma. Hepatology 2010;51:836-845.

28 Langsch S, Baumgartner U, Haemmig S, Schlup C, Schafer SC, Berezowska S, Rieger G, Dorn P, Tschan MP, Vassella E: miR-29b Mediates NF-kappaB Signaling in KRAS-Induced Non-Small Cell Lung Cancers. Cancer Res 2016;76:4160-4169.

29 Kirimura S, Kurata M, Nakagawa Y, Onishi I, Abe-Suzuki S, Abe S, Yamamoto K, Kitagawa M: Role of microRNA-29b in myelodysplastic syndromes during transformation to overt leukaemia. Pathology 2016;48:233-241.

-30 Ouyang YB, Xu L, Lu Y, Sun X, Yue S, Xiong XX, Giffard RG: Astrocyte-enriched miR-29a targets PUMA and reduces neuronal vulnerability to forebrain ischemia. Glia 2013;61:1784-1794.

31 Yang G, Song Y, Zhou X, Deng Y, Liu T, Weng G, Yu D, Pan S: MicroRNA-29c targets beta-site amyloid precursor protein-cleaving enzyme 1 and has a neuroprotective role in vitro and in vivo. Mol Med Rep 2015;12:3081-3088.

32 Han C, Chen X, Zhuang R, Xu M, Liu S, Li Q: miR-29a promotes myocardial cell apoptosis induced by high glucose through down-regulating IGF-1 Int J Clin Exp Med 2015;8:14352-14362.

-33 Arafa MH, Mohammad NS, Atteia HH, Abd-Elaziz HR: Protective effect of resveratrol against doxorubicininduced cardiac toxicity and fibrosis in male experimental rats. J Physiol Biochem 2014;70:701-711.

-34 Chen KC, Wang YS, Hu CY, Chang WC, Liao YC, Dai CY, Juo SH: OxLDL up-regulates microRNA-29b, leading to epigenetic modifications of MMP-2/MMP-9 genes: a novel mechanism for cardiovascular diseases. FASEB J 2011;25:1718-1728.

35 Maegdefessel L, Azuma J, Toh R, Merk DR, Deng A, Chin JT, Raaz U, Schoelmerich AM, Raiesdana A, Leeper NJ, McConnell MV, Dalman RL, Spin JM, Tsao PS: Inhibition of microRNA-29b reduces murine abdominal aortic aneurysm development. J Clin Invest 2012;122:497-506.

-36 Green DR, Reed JC: Mitochondria and apoptosis. Science 1998;281:1309-1312. 